EPJ Web of Conferences 101, 06062 (2015)

DOI: $10.1051 /$ epjconf/ 201510106062

(C) Owned by the authors, published by EDP Sciences, 2015

\title{
Tipsy pulsation of classical Cepheids - lessons from space photometry
}

\author{
L. Szabados ${ }^{1, a}$, N. R. Evans ${ }^{2}$, R. Szabó ${ }^{1}$, A. Derekas ${ }^{1,3}$, C. Cameron ${ }^{4}$, and the MOST team \\ 1 Konkoly Observatory, Budapest, Hungary \\ 2 SAO, Cambridge, USA \\ 3 ELTE Gothard Astrophysical Observatory, Szombathely, Hungary \\ 4 UBC, Vancouver, Canada
}

\begin{abstract}
Space photometric data of the Kepler Cepheid, V1154 Cygni, and those of SZ Tauri (MOST photometry) indicate that classical Cepheids are not strictly regular pulsators. Cycle-to-cycle period changes and variations in the shape of the light curve are revealed from the continuous photometry covering 6 cycles of SZ Tau pulsation and several hundred pulsation cycles of V1154 Cyg. To make the situation more interesting/complicated, the MOST light curve of RT Aurigae (a Cepheid pulsating in the fundamental mode) shows stellar oscillations in a highly repetitive manner.
\end{abstract}

\section{Introduction}

Classical Cepheids are the archetype of the regular radial pulsators. However, recently there are several pieces of photometric evidence at odds with this simplistic viewpoint. Folded light curves of small-amplitude Cepheids (possibly overtone pulsators) show wider scatter than their normal amplitude siblings performing fundamental-mode oscillations (see Fig. 1) [1]. Moreover, slightly excited nonradial modes were revealed in the pulsation of Magellanic Cepheids from the OGLE data base [2].

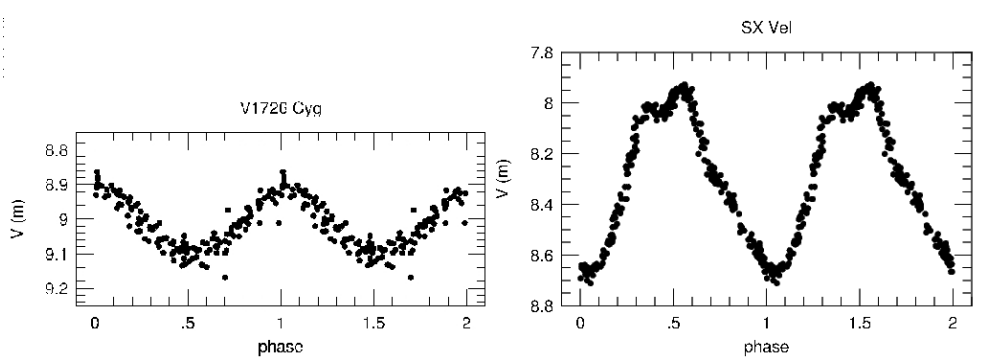

Fig. 1. The pulsation of low-amplitude Cepheids (e.g., V1726 Cyg, left) is more unstable than that of Cepheids pulsating in the fundamental mode (SX Vel, right) even when using the accurate (instantaneous) value of the pulsation period (data from [1])

The original Kepler field contains only one genuine Cepheid variable: V1154 Cygni [3]. The extremely precise photometry of Kepler space telescope was used for directly revealing fluctuations in the light curve and the pulsation period of V1154 Cygni [4].

a e-mail: szabados@konkoly.hu

This is an Open Access article distributed under the terms of the Creative Commons Attribution License 4.0, which permits unrestricted use, distribution, and reproduction in any medium, provided the original work is properly cited. 

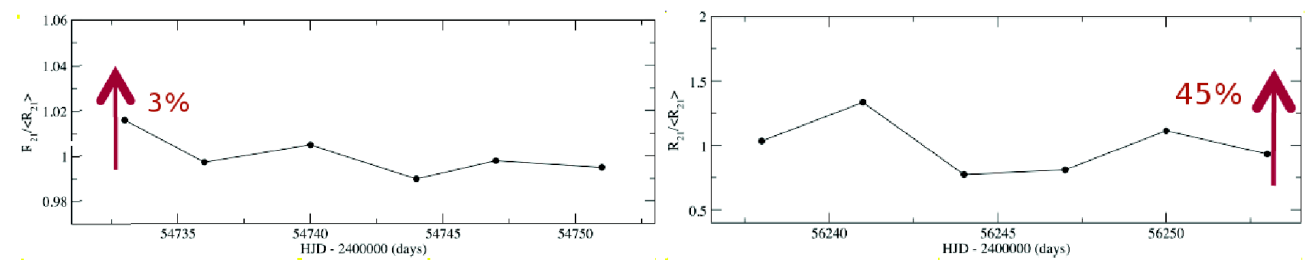

Fig. 2. Behaviour of the Fourier amplitude ratio, $R_{21}$ of RT Aur (left) and SZ Tau (right)

\section{MOST photometry of two Cepheids: SZ Tauri and RT Aurigae}

To confirm that the case of V1154 Cyg is not unique, a proposal was submitted for MOST observations of other bright Cepheids. Two targets were observed: the first-overtone pulsator SZ Tauri and RT Aurigae, a Cepheid pulsating in the fundamental mode.

The light curve of both Cepheids is unstable to some degree, and the overtone pulsator, SZ Tau shows a larger amplitude variance with pulsation cycle. For qualitative characterization of the jitter, the light curves have been decomposed using the Fourier parameters introduced by [5]. Both the amplitude ratio, $R_{21}$ (Fig. 2) and the phase parameters $\phi_{21}$ and $\phi_{31}$ fluctuate stronger in the overtone pulsator than in the fundamental-mode one.

In addition to the fluctuations in the shape of the light curve, the pulsation period also changes from cycle-to-cycle as testified by the $O-C$ diagrams. Again, the wobbling is stronger for the overtone pulsation [6].

\section{Conclusion}

The tipsy pulsation of classical Cepheids is an unexpected behaviour that calls for a theoretical explanation. An immediate hypothesis involves convection on the stellar surface [7].

Quite recently, a similar behaviour was found in the radial velocity phase curves of four Cepheids of rather different nature (pulsation mode and period): $\ell$ Car, QZ Nor, RS Pup, and V335 Pup [8].

Further accurate photometric observations of Cepheids are necessary. Future space photometry by Kepler K2, BRITE, Gaia, TESS, and PLATO will facilitate a better characterization of the jitter in the pulsation of classical Cepheids.

This research was supported by the European Space Agency (ESA), the Hungarian Space Office via the ESTEC Contract No. 4000106398/12/NL/KML, the Hungarian OTKA project K83790, the KTIA URKUT_10-1-20110019 grant, the Lendület-2009 Young Researchers Programme of the Hungarian Academy of Sciences, and the European Community's Seventh Framework Programme (FP7/2007-2013) under grant agreement no. 269194 (IRSES/ASK) and no. 312844 (SPACEINN). R.Sz. and A.D. were supported by the János Bolyai Research Scholarship of the Hungarian Academy of Sciences.

\section{References}

1. Berdnikov L. N., VizieR Online Data Catalog: Photoelectric observations of Cepheids in UBV(RI) (cyCat.2285....0B, 2008)

2. Moskalik, P. \& Kołaczkowski, Z. MNRAS 394, (2009) 1649

3. Szabó R., Szabados L., Ngeow C.-C., et al., MNRAS 413, (2011) 2709

4. Derekas A., Szabó Gy. M., Berdnikov L., et al., MNRAS 425, (2012) 1312

5. Simon N. R. \& Lee A. S., ApJ 248, (1981) 291

6. Evans N. R., Szabó R., Derekas A., et al., MNRAS 446, 4008 (2015)

7. Neilson H. R. \& Ignace R., A\&A 563, (2014) L4

8. Anderson R. I., A\&A 566, (2014) L10 